\title{
What is the growth mode of ultrathin Co films on $\operatorname{Pd}(001)$
}

\author{
Y.S. Shi* \\ Yancheng Teachers College, Yancheng, Jiangsu Province, 224002, China.
}

\begin{abstract}
Abstract: Thin Co films with varying thickness from 1 to 8 monolayers (ML) are epitaxially grown on $\mathrm{Pd}(001)$ and are studied in situ by using reflection high-energy electron diffraction (RHEED), scanning tunneling microscope (STM), low-energy electron diffraction (LEED), and magneto-optical Kerr effect (MOKE). The films take the layer-by-layer growth mode up to 3ML, and then switch to the 3dimensional growth mode afterward. For the as-grown films, MOKE measurements show that the easy axis of magnetism is in-plane and the interfacial $\mathrm{Pd}$ layer is magnetized. These results are confirmed and explained by density functional calculations.
\end{abstract}

Keywords: Magnetic anisotropy; Magnetic properties of mono layers; Structure and morphology.

\section{Introduction}

Growth and characterization of high quality ultrathin magnetic films on nonmagnetic substrates have been among the most active research subjects in the last three decades[1, 2, 3]. The broken symmetry and the reduced dimensionality due to the presence of surface or interface give rise various novel magnetic phenomena, such as perpendicular anisotropy, unusual magnetization and spin transport. $\mathrm{Co} / \mathrm{Pd}$ is a prototypical system in this realm and has attracted extensive attention due to the high magnetic susceptibility of $\mathrm{Pd}$ at the interface[4, 5, 6]. In addition, Co has rich structural phases, the growth mode of Co films on $\mathrm{Pd}$ in different growth conditions is also an

\footnotetext{
${ }^{*}$ Corresponding author

Email address: sys@yctc.edu.cn (Y.S. Shi)
} 
interesting issue. Co adopts the hexagonal close packed (hcp) structure in ambient, but it may change to the body centered cubic (bcc) or face centered cubic (fcc) structure when it grows on other substrates. Since there is a sizeable structural mismatch between $\mathrm{Co}$ and $\mathrm{Pd}$, it is questionable if the epitaxial growth of Co takes the layer-by-layer (2D-like) mode or multilayer island (3D-like) mode. On $\mathrm{Pd}(111)$, the growth of Co was found to change from the 2D-like mode to 3D-like mode when the Co film becomes thicker than two or three monolayers[7]. Co may form atomic chains and nanowires on $\operatorname{Pd}(110)$ in the submonolayer regime[8]. On $\operatorname{Pd}(001)$, it is still controversial if Co adopts the 2D-like mode or the 3D-like mode even from the onset.

The best fit between $\mathrm{Co}$ and $\mathrm{Pd}(001)$ is having Co in its bcc structure with a lattice constant of $a_{0}=0.283 \mathrm{~nm}$. After the Co lattice rotates by $45^{\circ}$, the lattice misfit between $\operatorname{Co}(001)$ and $\operatorname{Pd}(001)\left(a_{0}=0.389 \mathrm{~nm}\right)$ is $2.8 \%$. With a misfit like this, transition metals typically take the 2D-like growth mode in the ultrathin regime. However, from the low energy $\mathrm{He}^{+}$ion scattering (LEIS), X-ray photoelectron spectroscopy (XPS) and X-ray photoelectron diffraction (XPD) measurements, Giordano and co-workers declared that Co films grown on Pd by means of an electron beam evaporator form multiplelayer islands and their local structure is fcc-like, with the (001) plane parallel to the substrate surface[5]. In contrast, Meyerheim and coworkers reported that Co takes the face centered tetragonal (fct) structure and grows epitaxially in a layer-by-layer mode on the $\mathrm{Pd}(001)$ surface in their samples from surface X-ray diffraction measurements[9]. Since the structure dictates electronic and magnetic properties of the entire $\mathrm{Co} / \mathrm{Pd}$ sample, it is desirable to have the growth mode of Co more carefully examined[10]. In particular, one needs to use real-space techniques since information obtained through reciprocal space techniques such as LEIS and XPD is not adequate to reveal local structures without long-range coherent patterns in the real space.

In this work, we combined various techniques such as reflection high energy electron diffraction (RHEED), low energy electron diffraction (LEED), and scanning tunneling microscopy (STM) to investigate the growth of ultrathin Co films on $\mathrm{Pd}(001)$ in ultra high vacuum (UHV). In addition, we compared the morphologies of samples that are grown with either thermal deposition (TD) and pulsed laser deposition (PLD) so as to establish insights for the effect of atomic mobility on the growth mode. We find that Co takes the 2D-like growth mode when the thickness is less than three monolayers (MLs), regardless the growth condition. For Co films thicker than 10 MLs, 
the surface becomes extremely rough in STM images and the LEED pattern is also lost. However, RHEED oscillations can still be observed, which points out the possible ambiguity of using a single technique for the characterization of atomic geometries of metal thin films. Our finding is useful for understanding the controversies regarding the morphology of $\mathrm{Co} / \mathrm{Pd}(001)$ and paves a way for further studies of its electronic and magnetic properties.

\section{Experimental Methods}

The $\mathrm{Co} / \mathrm{Pd}$ films were grown at room temperature (RT), and characterized by in situ RHEED and STM. Magnetic properties were characterized by magneto-optical Kerr effect (MOKE) at RT and 70K. The experiments were performed in a multifunctional UHV system, equipped in addition to LEED, and Auger electron spectroscopy (AES). The base pressure in the UHV system was better than $5 \times 10^{-11}$ mbar and better than $2 \times 10^{-10}$ mbar during deposition. Prior to Co deposition, the clean $\operatorname{Pd}(001)$ surface was prepared by cycles of $2 \mathrm{keV}$ argon-ion bombardment at RT until no contamination was detectable by AES, followed by $1 \mathrm{kV}$ sputtering at $650 \mathrm{~K}$ for $15 \mathrm{~min}$. and final annealing at $950 \mathrm{~K}$ for $15 \mathrm{~min}$. The cleaning procedure was repeated until a sharp $(1 \times 1)$ LEED pattern was observed, and large atomically flat terraces were seen by STM.

The $\mathrm{Co} / \mathrm{Pd}(001)$ samples were prepared by thermal deposition, through evaporating Co from a Knudsen cell onto the clean $\operatorname{Pd}(001)$ surface. The deposition rate was about $0.2-0.3 / \mathrm{min}$, where the coverage is given in units of monolayers with respect to the $\operatorname{Pd}(001)$ surface atom density of $1.32 \times 10^{15} / \mathrm{cm}^{2}$. For pulsed laser deposition, a KrF excimer laser $(248 \mathrm{~nm}$ wavelength, $34 \mathrm{~ns}$ pulse length, repetition rate10 Hz, pulse energy $\approx 325$ $\mathrm{mJ}$ ) was focused on a Co target about $120 \mathrm{~mm}$ away from the sample surface. An average deposition rate between 0.1 and $0.3 \mathrm{ML} / \mathrm{min}$ was achieved, comparable to the deposition rate for TD. In all cases the sample was kept at room temperature. The film thickness was cross-checked by RHEED oscillations and by STM, and estimated with an accuracy of $0.1 \mathrm{ML}$. The Co films were grown either in a uniform sample or in wedge shape samples that have a continuous variation of film thickness. The wedge-like Co films were prepared by moving the $\operatorname{Pd}(001)$ substrate behind a fixed shutter during the Co deposition. Using this method we made around twenty steps in one sample, which means that we can get about twenty different thicknesses in one 
sample that is about $1 \mathrm{~cm}$ wide. Several wedge samples of $\mathrm{Co} / \mathrm{Pd}(001)$ were grown in the course of study.

\subsection{Results and Discussions}

The growth of Co on a $\operatorname{Pd}(001)$ substrate was first investigated in situ by the development of RHEED patterns as a function of the Co coverage. The growth mode of the Co film was determined by following the intensity changes of the $(1,0),(0,0)$, and $(-1,0)$ diffraction spots during the growth. According to the interlayer spacing of the fcc Co $(0.177 \mathrm{~nm})$, the RHEED scattering angle was set to correspond to the second anti-Bragg reflection. As shown in Fig.1 for the RHEED intensity measured at the specular spot as a function of Co coverage, three well-defined RHEED oscillations are clearly seen at the initial stage of Co deposition. This indicates that the initial growth of $\mathrm{Co}$ on $\mathrm{Pd}(001)$ is indeed in the layer-by-layer mode. The fast decay of the RHEED oscillation amplitudes, however, implies that the surface roughening happens at very early stage, which partly explains why the 3Dlike growth mode was proposed in other studies[5]. By assuming that clear RHEED oscillation is an indication of layer-by-layer growth, one may also claim the 2D-like growth mode for $\mathrm{Co}$ on $\mathrm{Pd}(001)$ since we can even see the $9^{\text {th }}$ RHEED oscillations in the amplified RHEED spectrum (shown in the inset in Fig.1) [9].

As a verdict for this controversy, Figure 2 shows a set of STM images taken at RT. Clearly, the development of complex surface morphology can be seen for the $\mathrm{Co} / \mathrm{Pd}(001)$ system when the Co film is thicker than 3 MLs. In order to have an internal calibration of the vertical heights, some welldefined monatomic steps of the clean $\mathrm{Pd}(001)$ surface are shown in Fig.2(a). The rest of figures show the evolution of surface morphology caused by Co continuous deposition and hence the increase of Co coverage. In the submonolayer region, as shown in Fig.2(b), only monatomic high-Co islands are found on the $\operatorname{Pd}(001)$ substrate when the Co coverage is $0.5 \mathrm{ML}$. The inset of Fig.2(b) shows a line profile that clearly indicates only monatomic-high Co film. In addition, it is obvious to see in this figure that such a 2D-like film actually consists of a large amount of randomly distributed monolayer thick patches. This result is very similar to $\mathrm{Fe} / \mathrm{Pd}(001)$ for which the surface morphology in the submonolayer regime is characterized by a random distribution of Fe patches on the $\mathrm{Pd}(001)$ surface[11, 12, 13]. Up to 0.7 ML, no bilayer Co cluster is found in STM images (not shown here). When the Co coverage reaches $0.8 \mathrm{ML}$, an overall flat monolayer Co film is still seen in 
Fig.2(c), but a few patches start developing the second layer of Co (bright dots) despite the existence of uncovered areas (black dots) of the clean $\mathrm{Pd}$ surface. When Co coverage increases further from $1 \mathrm{ML}$ to $2 \mathrm{ML}$, the overall feature of the second layer growth of Co is similar to that of the first layer. One may still call that Co grows in a 2D-like growth mode at this stage, since the STM images in Fig.2(d) for 1.0 ML Co and Fig.2(e) for 1.8-ML Co are similar to their counterparts in Fig.2(c). The surface morphology apparently becomes rougher, starting from the third layer of Co as can be clearly seen in Fig.2(f). Finally, STM images for 6 ML Co in Fig.2(g) and 10 ML Co in Fig.2(h) show the presence of very rough Co islands with varying heights. Accordingly, the RHEED oscillation fades away. To see if the enhanced mobility of Co atoms in PLD changes the growth mode, we present the STM images of PLD Co/Pd samples with 0.5 ML and 1.5 ML Co in Fig.3(a) and (b). No obvious difference can be seen from those in Fig.2. It means that the morphologies of Co films on $\mathrm{Pd}(001)$ by TD and PLD are almost the same.

Fig.4 shows the LEED pattern of the Co films grown on $\operatorname{Pd}(001)$ with different thicknesses. The clean $\operatorname{Pd}(001)$ substrate shows a sharp $(1 \times 1)$ diffraction pattern in Fig.4(a). From the other LEED patterns, the $p(1 \times$ 1) structure can only be seen for samples with Co thinner than 3ML, as shown in Fig.4(b)-(e). This thickness range corresponds well to the 2Dlike growth of Co as we discussed above according to the STM images and RHEED oscillations. Since Co atoms in this pesudomorphic fct structure have large stress, further increase of Co thickness quickly leads to blur LEED patterns with a strong diffuse background as seen in Fig.4(f) for 6 ML Co on $\mathrm{Pd}(001)$. For $10 \mathrm{ML}$ Co film on $\mathrm{Pd}(001)$, there is no visible LEED pattern. This also indicates the eventual development of a 3D-like growth mode of Co above a thickness of 3ML. It appears that LEED is more sensitive for the identification of the growth mode of $\mathrm{Co} / \mathrm{Pd}(001)$ since it requires the coherence of electron diffraction in two directions. Fig.4(g) and (h) show the RHEED patterns of the $10 \mathrm{ML}$ Co film on $\mathrm{Pd}(001)$. When the e-beam is along the [110] direction of the Pd substrate the RHEED pattern is a tetragonal plus center spot. In contrast, the RHEED pattern is a tetragonal when ebeam is along [100] direction. While this also shows some inhomogeneity of the structure, the information is vague for the determination of actual atomic structures. Therefore, we may say that small RHEED oscillations peaks cannot be used as an evidence of the 2D-like growth mode. Finally, our MOKE measurements indicate that the $\mathrm{Co} / \mathrm{Pd}(001)$ is ferromagnetic and the easy axis is in-plane in the entire thickness rage. As observed in previous 
studies, the Kerr signal increases almost linearly with the Co thickness[14, 15, 16]. A nonzero magnetization is extrapolated when the Co thickness approaches to zero, due to the induced magnetization in the interfacial $\mathrm{Pd}$ layers[17].

\section{3. summary}

In summary, Co ultrathin films were grown on $\operatorname{Pd}(001)$ and investigated by RHEED, LEED, STM, and MOKE. The growth appears to be smooth with the 2D-like mode for a Co coverage up to 3ML. Above this coverage the 3D-like growth is observed. Room-temperature-grown Co film shows inplane magnetization after immediately measurement. We compared samples grown with either TD or PLD method, and found that their morphologies and magnetic properties are almost the same. This indicates that the growth mode is mainly governed by the lattice mismatch or lattice strain.

\section{Acknowledgments:}

This work was supported by the National Science Foundation of China, under a contract number: 11174243.

\section{References}

[1] J.R. Cerda, P.L. de Andress, A. Cebollada, R. Miranda, E. Navas, P. Schuster, C.M. Schneider, and J. Kirschner, J. Phys. Condens. Matter5, 2055 (1993)

[2] F. J. Himpsel, J. E. Ortega, G. J. Mankey, and R. F. Willis, Adv. Phys. 47, 511 (1998)

[3] J.A.C. Bland, and B. Heinrich, in Ultrathin Magnetic Structures (Springer-Verlag Berlin, Heidelberg, 1994)

[4] S.Blugel, Europhysics Letters 7, 743 (1988)

[5] H. Giordano, A. Atrei, G. Rovida, M. Torrini, and U. Bardi, J. Electron. Spectrosc. Relat. Phenom. 76, 455 (1995), H. Giordano, A. Atrei, M. Torrini, U. Bardi, M. Gleeson, and C.Barnes, Phys. Rev. B 54, 11762 (1996) 
[6] B. N. Engel, C. D. England, R. A. Van Leeuwen, M. H. Wiedmann, and C. M. Falco, Phys. Rev. Lett. 67, 1910 (1991)

[7] Jonggeol Kim, Jeong-Won Lee, Jong-Ryul Jeong, Sang-Koog Kim, and Sung-Chul Shin, J. Appl. Phys. 89, 7147 (2001)

[8] L. Yan, M. Przybylski, Y. Lu, W. H. Wang, J. Barthel, and J. Kirschner, Appl. Phys. Lett. 86, 102503 (2005)

[9] H.L. Meyerheim, V. Stepanyuk, A.L. Klavsyuk, E. Soyka, and J. Kirschner, Phys. Rev. B 72, 113403 (2005)

[10] J. Dorantes-Davila and G. M. Pastor, Phys. Rev. Lett. 81, 208 (1998)

[11] C. Liu and S. D. Bader, J. Appl. Phys. 67, 5758 (1990)

[12] X. F. Jin, J. Barthel, J. Shen, S. S. Manoharan, and J. Kirschner, Phys. Rev. B 60, 11809 (1999)

[13] C. Liu and S. D. Bader, J. Magn. Magn. Mater. 93, (1991) 307; Phys.Rev. B 44, 2205 (1991)

[14] Z.Q. Qiu, J. Pearson, and S.D. Bader, Phys. Rev. B 46, 8195 (1992)

[15] Z.Q. Qiu and S.D. Bader, J. Magn. Magn. Mater. 200, 664 (1999)

[16] J. W. Lee, J. R. Jeong, S. C. Shin, J. Kim, and S. K. Kim, Phys. Rev. B 66, 172409 (2002)

[17] Y.S. Shi, M. Przybylski, L. Yan, J. Barthel, and J. Kirschner (in preparation) 


\section{Figure Captions}

Figure 1 RHEED intensity oscillations measured at the specular spot during the growth of $\mathrm{Co}$ on $\mathrm{Pd}(001)$. The period of the oscillations corresponds to the time required to form one atomic layer.

Figure $2 \mathrm{~A}$ set of STM images as a function of Co coverage on $\mathrm{Pd}(001)$. (a) Clean $\operatorname{Pd}(001)$ surface $\left(400 \times 400 \mathrm{~nm}^{2}\right)$; (b)-(h) corresponds to the Co film thickness at 0.5, 0.8, 1.0,1.8, 2.7, 6.010.0 ML. The scan of (b)-(h) images is $100 \times 100 \mathrm{~nm}^{2}$.

Figure 3 STM images for (a) 0.5 ML PLD-grown Co on Pd(001) $(100 \times$ $\left.100 \mathrm{~nm}^{2}\right)$; (b)1.5 ML PLD-grown Co on $\operatorname{Pd}(001)\left(100 \times 100 \mathrm{~nm}^{2}\right)$.

Figure 4 LEED patterns of $\mathrm{Co}$ on $\operatorname{Pd}(001)$ : (a) Clean $\operatorname{Pd}(001)$ surface; (b) $0.5 \mathrm{ML}$; (c) $1 \mathrm{ML}$; (d) $1.8 \mathrm{ML}$; (e) $2.7 \mathrm{ML}$; (f) 6ML. RHEED patterns of 10ML Co film on Pd(001): (g) e-beam \| 110; (h) e-beam \| 100. 
Fig.1

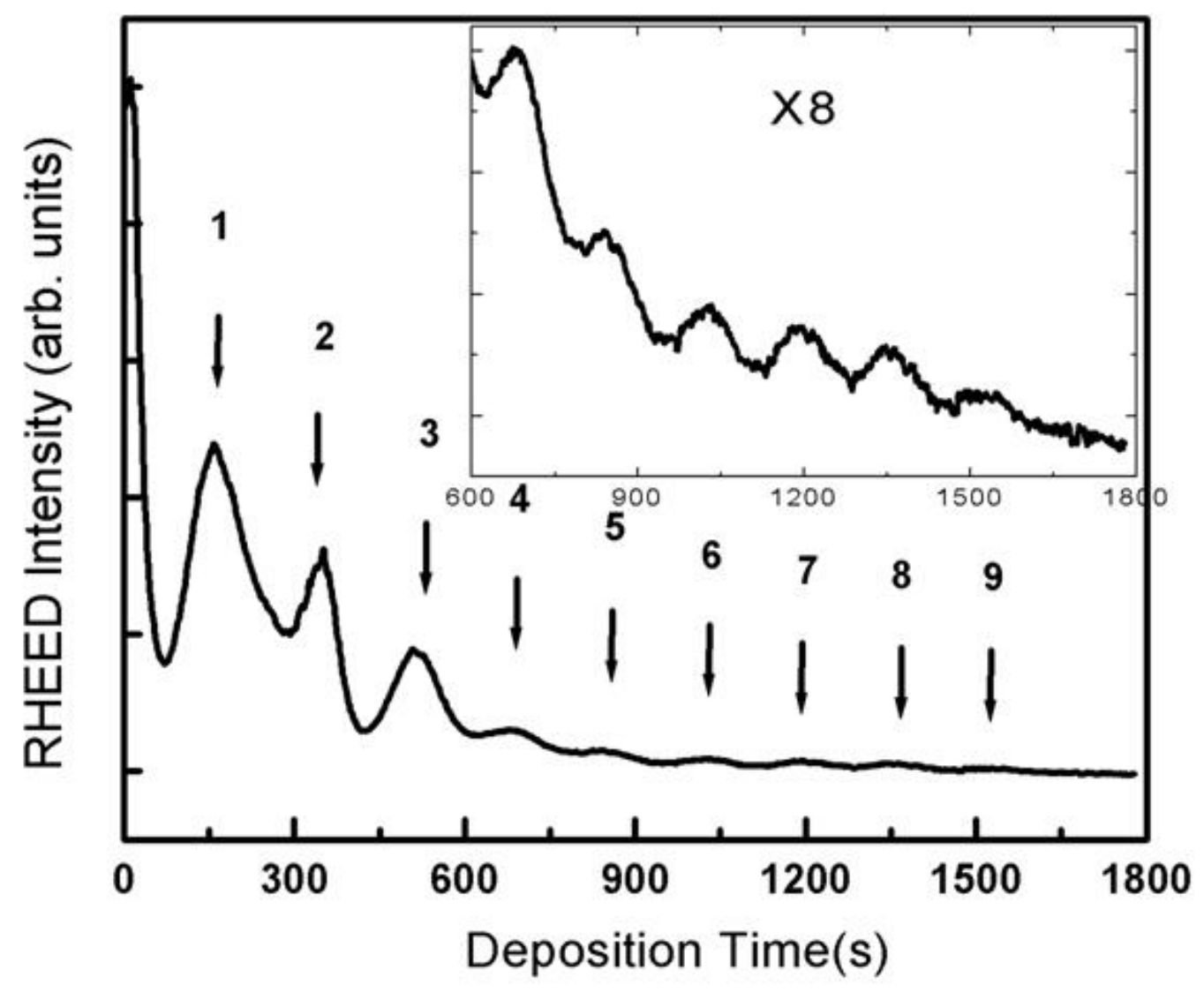


Fig.2
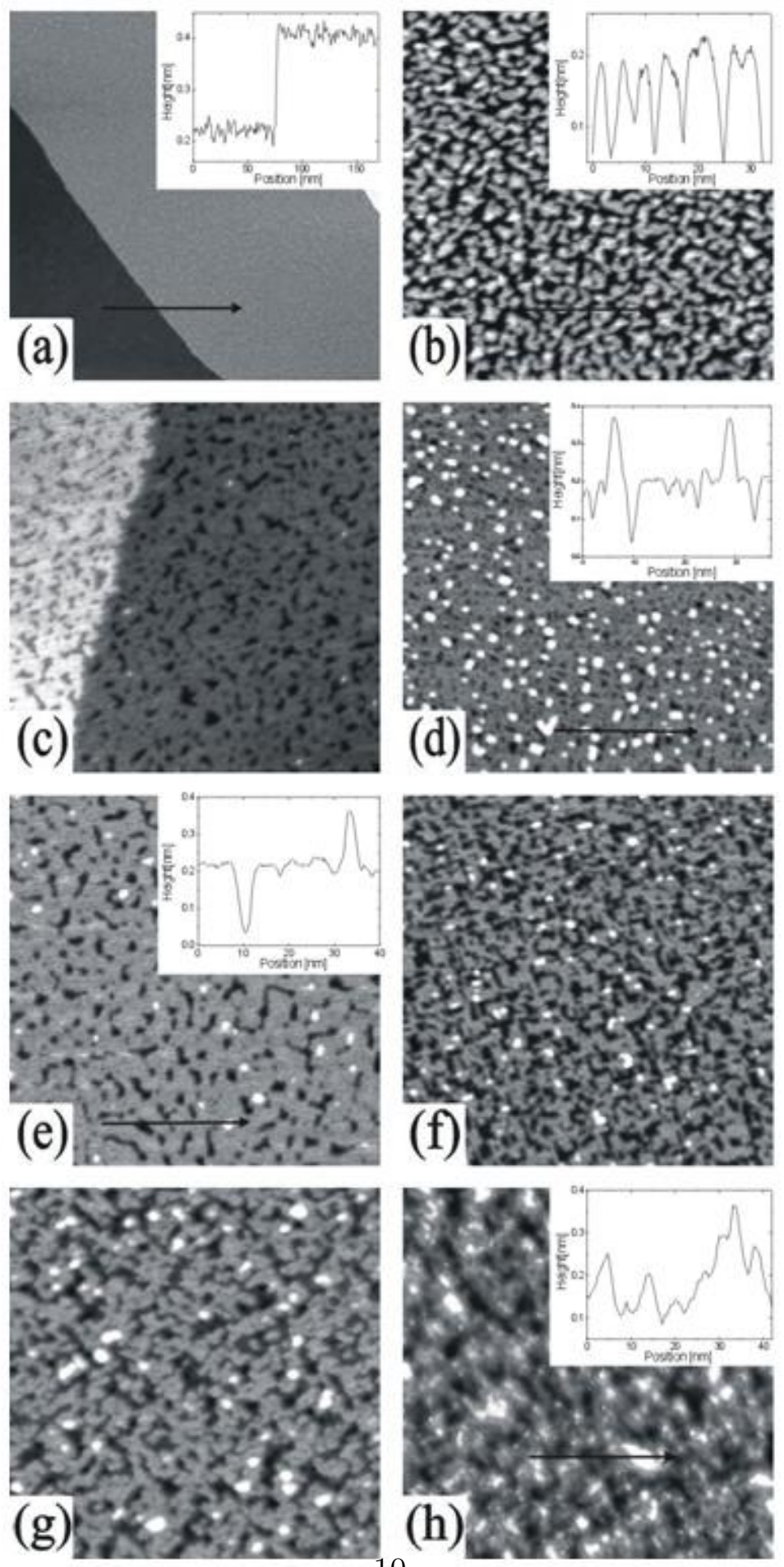
Fig.3
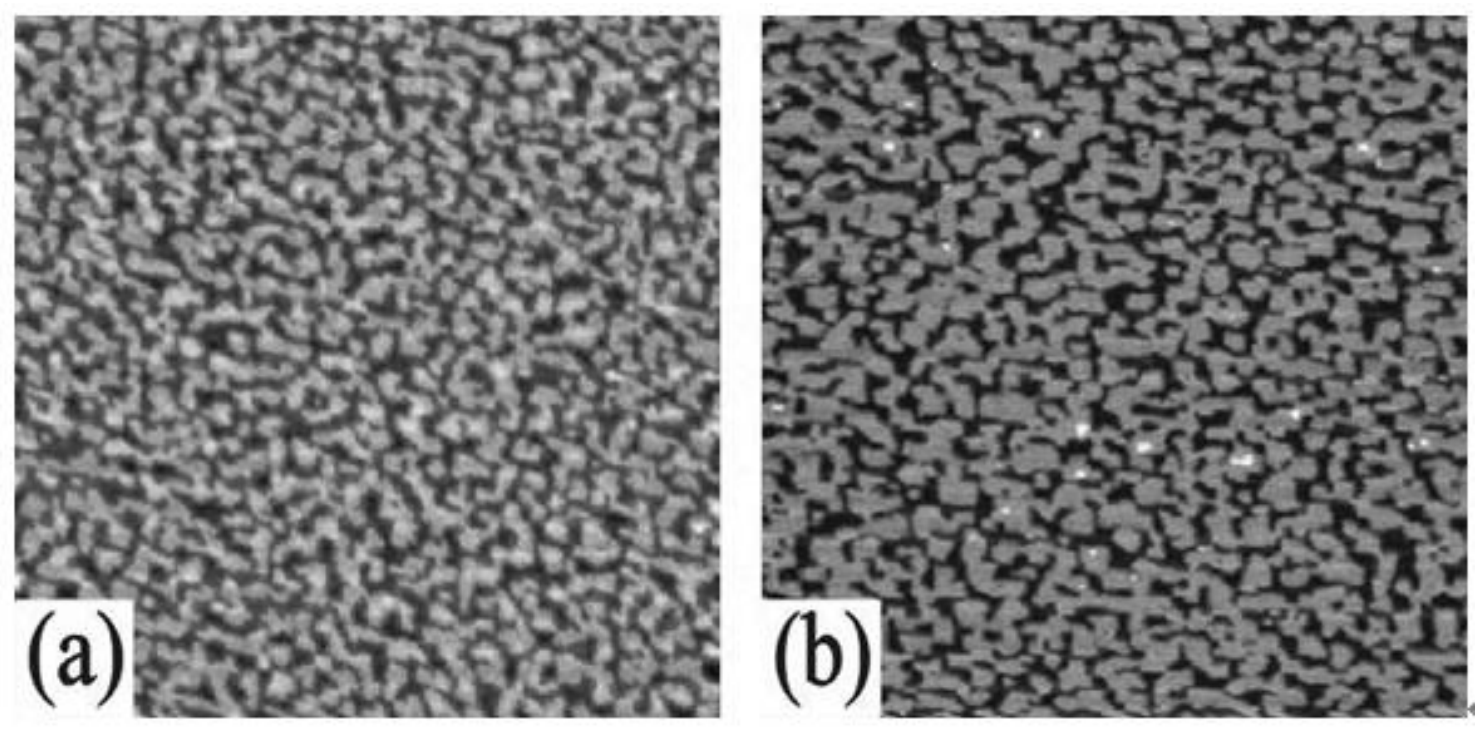
Fig.4
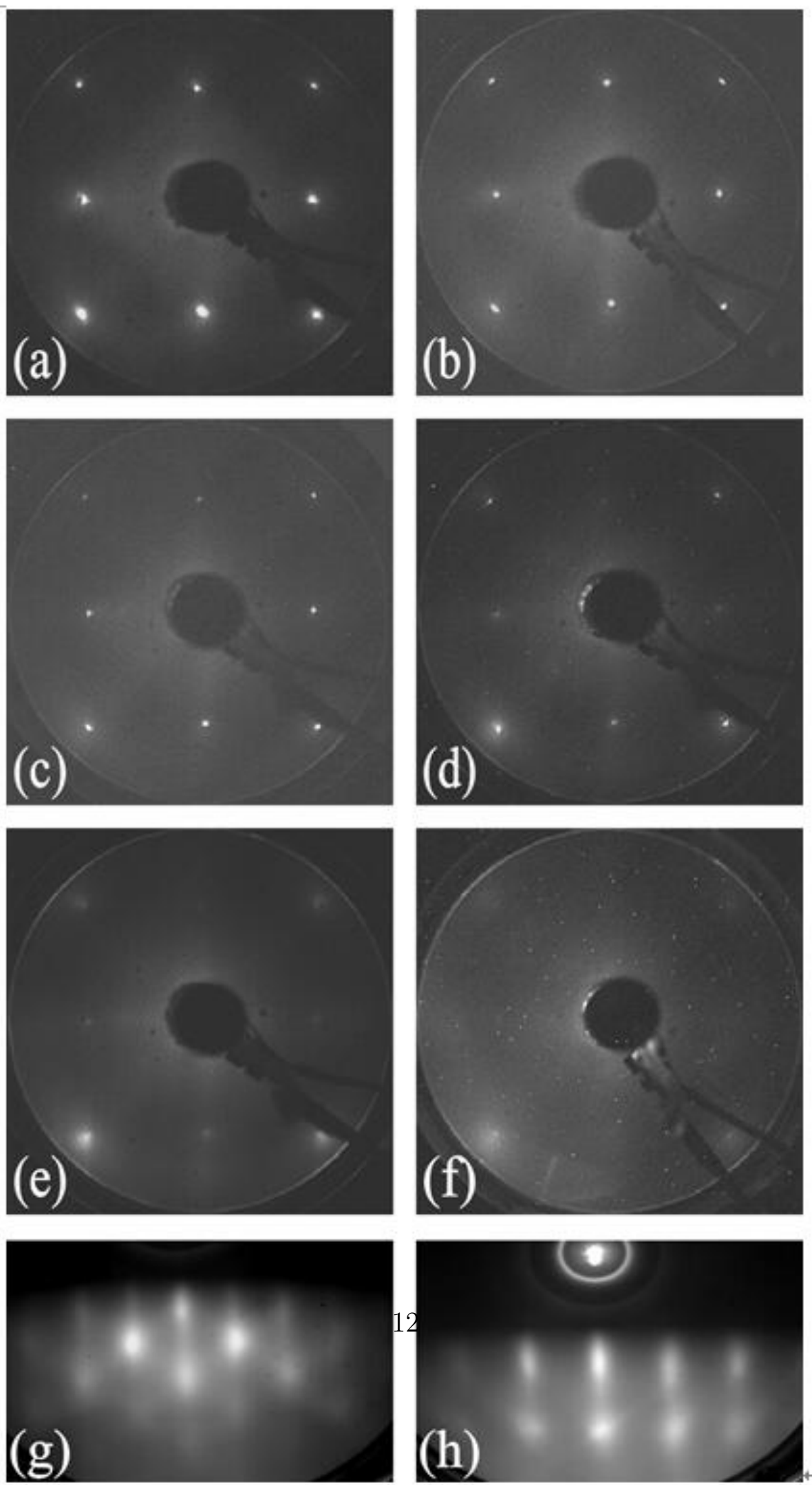